\title{
Aberrant gene methylation in non-neoplastic mucosa as a predictive marker of ulcerative colitis-associated CRC
}

\author{
Marco Scarpa ${ }^{1, *}$, Melania Scarpa $a^{1, *}$, Ignazio Castagliuolo ${ }^{2}$, Francesca Erroi ${ }^{3}$, \\ Andromachi Kotsafti ${ }^{1}$, Silvia Basato ${ }^{3}$, Paola Brun ${ }^{2}$, Renata D'Incà ${ }^{3}$, Massimo Rugge ${ }^{4}$, \\ Imerio Angriman ${ }^{3, *}$, Carlo Castoro ${ }^{1, *}$ \\ ${ }^{1}$ Surgical Oncology Unit, Veneto Institute of Oncology IOV - IRCCS, Padova, Italy \\ ${ }^{2}$ Department of Molecular Medicine, University of Padova, Padova, Italy \\ ${ }^{3}$ Department of Surgery Oncology and Gastroenterology DISCOG, University of Padova, Padova, Italy \\ ${ }^{4}$ Department of Medicine, University of Padova, Padova, Italy \\ *These authors have contributed equally to this work \\ Correspondence to: Marco Scarpa, e-mail: marcoscarpa73@yahoo.it \\ Keywords: biomarker, ulcerative colitis, colorectal cancer, promoter methylation, APC \\ Received: October 08, 2015 \\ Accepted: January 23, 2016 \\ Published: February 04, 2016
}

\section{ABSTRACT}

Background Promoter: hypermethylation plays a major role in cancer through transcriptional silencing of critical genes. The aim of our study is to evaluate the methylation status of these genes in the colonic mucosa without dysplasia or adenocarcinoma at the different steps of sporadic and UC-related carcinogenesis and to investigate the possible role of genomic methylation as a marker of CRC.

Results: The expression of Dnmts 1 and 3A was significantly increased in UC-related carcinogenesis compared to non inflammatory colorectal carcinogenesis. In non-neoplastic colonic mucosa, the number of methylated genes resulted significantly higher in patients with CRC and in those with UC-related CRC compared to the HC and UC patients and patients with dysplastic lesion of the colon. The number of methylated genes in non-neoplastic colonic mucosa predicted the presence of CRC with good accuracy either in non inflammatory and inflammatory related CRC.

Methods: Colonic mucosal samples were collected from healthy subjects $(\mathrm{HC})(n=30)$ and from patients with ulcerative colitis (UC) $(n=29)$, UC and dysplasia $(n=14)$, UC and cancer $(n=10)$, dysplastic adenoma $(n=14)$, and colon adenocarcinoma $(n=10)$. DNA methyltransferases-1, -3a, -3b, mRNA expression were quantified by real time qRT-PCR. The methylation status of CDH13, APC, MLH1, MGMT1 and RUNX3 gene promoters was assessed by methylation-specific PCR.

Conclusions: Methylation status of APC, CDH13, MGMT, MLH1 and RUNX3 in the non-neoplastic mucosa may be used as a marker of CRC: these preliminary results could allow for the adjustment of a patient's surveillance interval and to select UC patients who should undergo intensive surveillance.

\section{INTRODUCTION}

Ulcerative colitis (UC) is a chronic inflammatory disorder involving the rectum and the colon that expose patients to an increased risk of colorectal cancer (CRC) [1]. In fact, in patients with UC, the cumulative risk of CRC is approximately $8 \%$ twenty years after the diagnosis and the cumulative rate of dysplasia is at least $25 \%$ $[2,3,4]$. Several independent risk factors for malignancy in UC patients have been identified such as duration of disease, extent of inflammation, history of concurrent primary sclerosing cholangitis and family history of CRC [5]. Pre-malignant histological alterations in UC patients are broadly referred to as dysplasia, rather than adenoma, since dysplasia is frequently not polypoid $[6,7]$.

Therefore, patients with UC at moderate or high risk for CRC are advised to undergo surveillance colonoscopy and biopsy, every 1 to 3 years accordingly to 
different guidelines, where multiple random biopsies are histologically evaluated for the presence of pre-cancerous changes (colorectal dysplasia) or CRC [8-10]. CRC screening in healthy subject is based on occult blood in the stool but in patients with UC the screening imply yearly colonoscopy with a significant higher grade of invasiveness. This highlights the need to identify markers for UC-related cancer that can be detected in the non neoplastic areas of the colon. The latter component would be of particular benefit because dysplasia or CRC may be difficult to identify endoscopically in UC, and a test that is not reliant on biopsies taken from dysplastic areas might be carried out by a simple sigmoidoscopy and thus it would be of particular interest as an adjuvant screening tool [11].

An altered methylation pattern in cancer cell genomes is a well recognized characteristic of tumor cells, and specific aberrant methylation events take place during the early stages of colorectal carcinogenesis leading to profound modifications in gene expression [12]. In particular, altered methylation status of RUNX3, MINT1, and COX-2 had been observed in the non-neoplastic sections of UC-related CRC colons as compared with that in the UC controls [11]. The aberrant methylation of $\mathrm{H}$-cadherin (CDH13) commencing at an early stage of colorectal tumorigenesis frequently silences, in fact, the expression of this tumor suppressor gene in colorectal adenomas and cancers [13]. Besides germ-line mutations associated with hereditary familial adenomatous polyposis and somatic mutations in sporadic colorectal tumors, hypermethylation also provides an important mechanism underlying impaired APC function [14]. Moreover, the hypermethylation of the $\mathrm{CpG}$ island within the DNA-repair protein O-6-methylguanine-DNA-methyltransferase (MGMT) gene [15] and in the MLH1 gene is associated with a reduced gene expression observed in the majority of sporadic primary CRC cancers with microsatellite instability [16]. Finally, RUNX3 hypermethylation decreases TGF- $\beta / \mathrm{BMP}$ signalling in gastrointestinal cancer cells [17].

Therefore, the aim of our study is to evaluate the methylation status of these genes in the non-neoplastic (without dysplasia or adenocarcinoma) colonic mucosa at different steps of non inflammatory and UC-related carcinogenesis and to investigate the possible role of genomic methylation in the non-neoplastic colonic mucosa as a marker of UC-related CRC.

\section{RESULTS}

\section{Patients characteristics}

The promoter methylation status of five genes known to be associated with early stages of colon carcinogenesis was assessed in the colonic mucosa of 107 patients (Table 1). They consisted of 54 patients representative of non-inflammatory colon carcinogenesis pathway
(30 healthy controls, 14 with adenoma and dysplasia, and 10 with cancer) and 53 patients with UC-related carcinogenesis (29 with UC, 14 with UC and dysplasia, 10 with UC and cancer).

\section{DNA methyltransferase mRNA expression along colonic carcinogenesis pathway}

To understand how the inflammatory condition may influence the genomic methylation status, Dnmt-1, $-3 a$, and $-3 b$ mRNA expression was quantified in the different steps of non inflammatory and inflammatory carcinogenesis in colonic tissue without lesions. As shown in Figure 1, the expression of all three Dnmts tended to increase progressively along the carcinogenesis pathways; moreover a significant higher expression was detected in the UC-related carcinogenesis compared to the non-inflammatory type. Dnmt-1: healthy control (HC) vs UC patients, $p<0.001$; adenoma vs UC and dysplasia, $p=0.09$; adenocarcinoma vs UC and cancer, $p=0.008$ (Figure 1A). Dnmt-3A: HC vs UC patients, $p<0.001$; adenoma vs UC and dysplasia, $p=0.001$; adenocarcinoma vs UC and cancer, $p=0.012$ (Figure 1B). Dnmt-3B: HC vs UC patients, $p=0.029$; adenoma vs UC and dysplasia, $p=0.288$; adenocarcinoma vs UC and cancer, $p=0.321$ (Figure 1C).

\section{Methylation status of gene promoters involved in the early stages of colon carcinogenesis}

As shown in Figure 2A, in colonic tissue without lesions, the number of methylated genes resulted significantly higher in patients with adenocarcinoma than in $\mathrm{HC}$ and in those with adenomas $(p=0.03)$. Similarly, the number of methylated genes resulted significantly higher in patients with UC-related adenocarcinoma than in those with UC and in those with UC and dysplasia $(p=0.02)$. In colonic tissue without lesions, as shown in Figure $2 \mathrm{~B}$, the promoter regions of APC was significantly more frequently methylated in patients with UC and cancer compared to patients with non inflammatory adenocarcinoma $(p=0.05)$. Moreover, as shown in Figure 2C, the promoter regions of $\mathrm{CDH} 13$ was significantly more frequently methylated in patients with $\mathrm{UC}$ and dysplasia compared to those with inflammatory adenoma $(p=0.01)$. In contrast, the frequency of methylation of the MGMT and MLH1 promoters was not significantly different between the UC-related and the not-inflammatory carcinogenesis pathways (Figure 2D and 2E). On the contrary, as shown in Figure 2F, the promoter regions of RUNX3 was significantly more frequently methylated in patients with UC and cancer compared to patients with non inflammatory adenocarcinoma $(p=0.01)$. 
Table 1: Real-time qPCR and methylation-specific PCR primers

\section{A. Real-Time qPCR primers}

\begin{tabular}{|c|c|c|c|c|}
\hline \multicolumn{1}{c}{ Gene } & NCBI ref seq & Sequence 5'-->3' & Ta. ${ }^{\circ} \mathbf{C}$ & Amplicon. bp \\
\hline Dnmt1 & NM_001130823 & fw TACCTGGACGACCCTGACCTC & 60 & 103 \\
\hline & & rv CGTTGGCATCAAAGATGGACA & & \\
\hline Dnmt3a & NM_175629 & fw GACAAGAATGCCACCAAAGC & 60 & 190 \\
\hline & & rv CGTCTCCGAACCACATGAC & & \\
\hline Dnmt3b & NM_006892 & fw GGCAAGTTCTCCGAGGTCTCTG & 60 & 113 \\
\hline & & rv TGGTACATGGCTTTTCGATAGGA & & \\
\hline
\end{tabular}

B. Methylation specific PCR primers

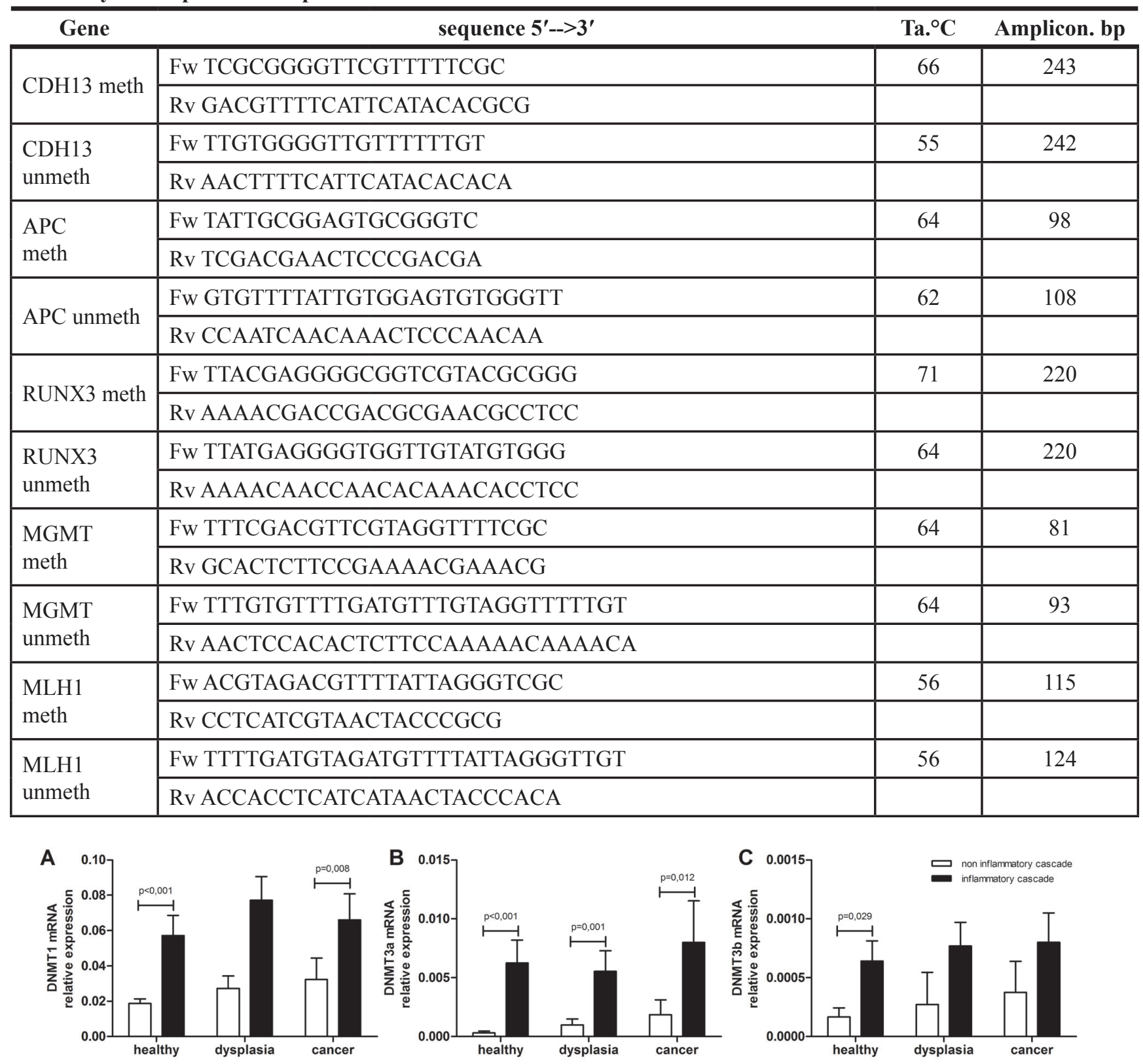

Figure 1: DNA methyltransferases mRNA levels in colon carcinogenesis. (A) DNMT1 (B) DNMT3a and (C) DNMT3b mRNA levels were quantified by Real Time qPCR in the colonic mucosa specimens of patients with non-inflammatory colon carcinogenesis and UC-related colon carcinogenesis; mRNA mean levels \pm SEM in the different patients groups are shown. Kruskal-Wallis ANOVA test was performed to compare multiple groups. HC. healthy control; DYS. dysplasia and adenoma; UC. ulcerative colitis. 


\section{Accuracy of the number of methylated genes in predicting the presence of cancer in the colon}

To assess the accuracy of the number of methylated genes as predictor of dysplasia and cancer in inflamed and in normal colon we performed ROC curve analysis. As shown in Figure 3A, in UCrelated carcinogenesis the number of methylated genes in colonic mucosa without lesions predicted the presence of dysplasia or cancer in UC patients with low accuracy (AUC $=0.6895 \% \mathrm{CI}=0.54-0.80$; $p=0.01833$ ). However, the number of methylated genes in colonic mucosa without lesions predicted the presence of cancer in patients with UC with good accuracy $(\mathrm{AUC}=0.7795 \% \mathrm{CI}=0.63-0.88 ; p=0.011)$ (Figure 3B). Indeed, over 2 methylated genes in non-neoplastic mucosa predicted the presence of CRC in patients with UC with a sensitivity of $57.1 \%(95 \% \mathrm{CI}=18.8-89.6)$ and a specificity of $93.1(95 \% \mathrm{CI}=80.9-98.5)$. Similarly, as shown in Figure 3C, in non inflammatory carcinogenesis the number of methylated genes in colonic mucosa without lesions predicted the presence of adenoma or cancer with poor accuracy (AUC $=0.6095 \% \mathrm{CI}=0.46-0.73$; $p=0.1833$ ). Nevertheless, the number of methylated genes in colonic mucosa without lesions predicted the presence of cancer in patients without UC with good accuracy $(\mathrm{AUC}=0.7595 \% \mathrm{CI}=0.62-0.85 ; p=0.008)($ Figure 3D) .

\section{Number of methylated genes as risk factor for $\mathrm{CRC}$ in $\mathrm{UC}$}

To assess the possible interaction with other known predictors of CRC we tested the number of methylated genes with the grade of histological inflammation, the duration of the disease and the familial history of CRC. As shown in Figure 4A, inflammatory grade as measured by Floren score on the same mucosal sample showed a good accuracy in predicting UC-related CRC (AUC $=0.77$
A

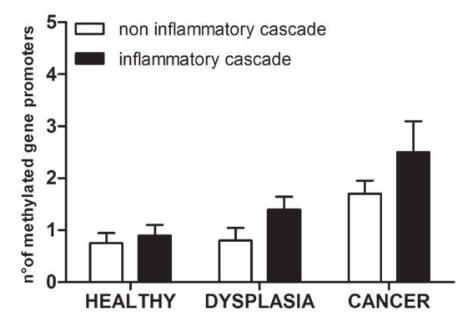

C

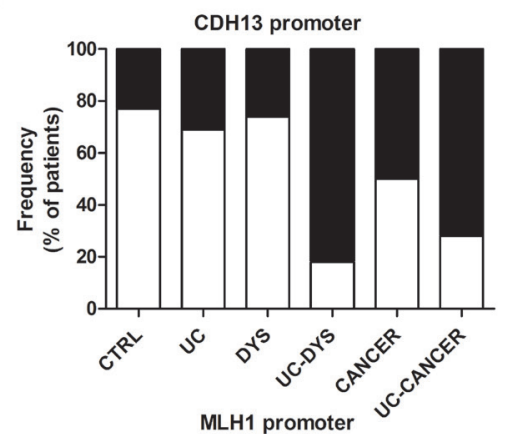

E

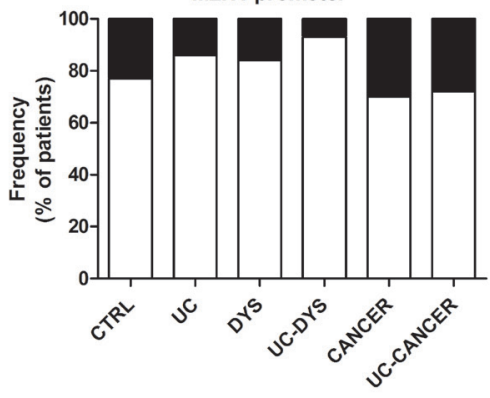

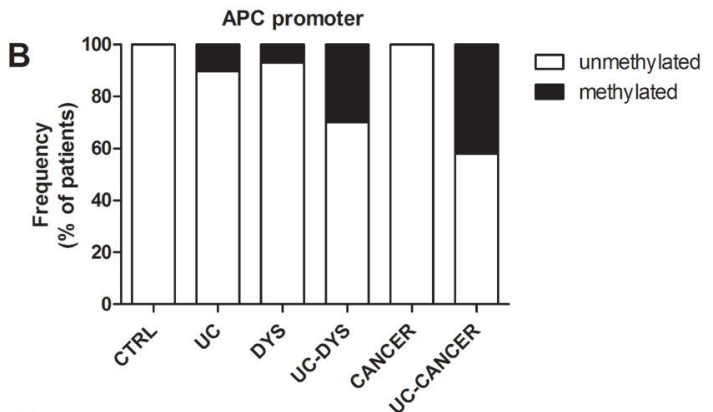

D

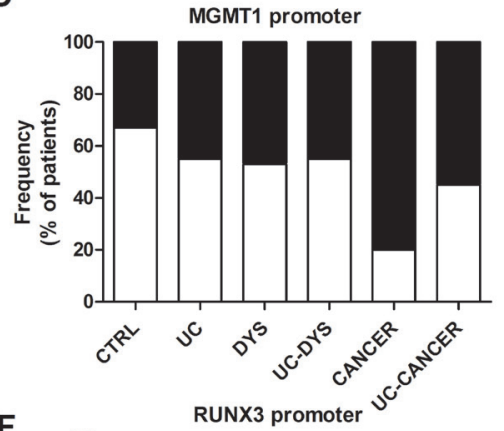

$\mathbf{F}$

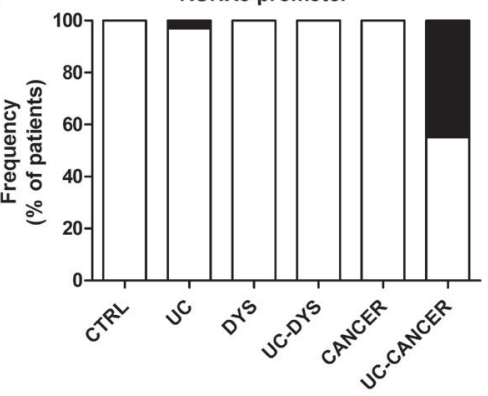

Figure 2: Methylation status of gene promoters involved in the early stages of colon carcinogenesis. (A) The frequency of total methylation. defined as the sum of the methylated genes detected (range 0-5) among the different patients groups is shown. (B, C, D, E, F) The methylation status of APC. CDH13. MGMT. MLH1 and RUNX3 gene promoters was assessed by methylation specific-PCR of colonic mucosa specimens with no neoplastic lesion derived from patients with non-inflammatory colon carcinogenesis and UC-related colon carcinogenesis. The frequency of methylation of each gene among the different patients groups is shown. HC. healthy control; DYS. dysplasia and adenoma; UC. ulcerative colitis. 
95\% CI $=0.64-0.88 ; p<0.001)$. Moreover, as shown in Figure 4B, disease duration of patients showed a low accuracy in predicting UC-related CRC (AUC $=0.6595 \%$ $\mathrm{CI}=0.49-0.79 ; p<0.151)$. Similarly, familial history of CRC showed a odd ratio of $0.578(95 \% \mathrm{CI}=0.059-5.631)$ for CRC in UC. As shown in Figure 4C, multiple logistic regression analysis showed that both Floren score and methylation score, but not disease duration and familial history of CRC, were independent predictors of UC-related CRC.

\section{DISCUSSION}

UC is a chronic inflammatory disorder that shows increased risk of CRC [1] with a cumulative
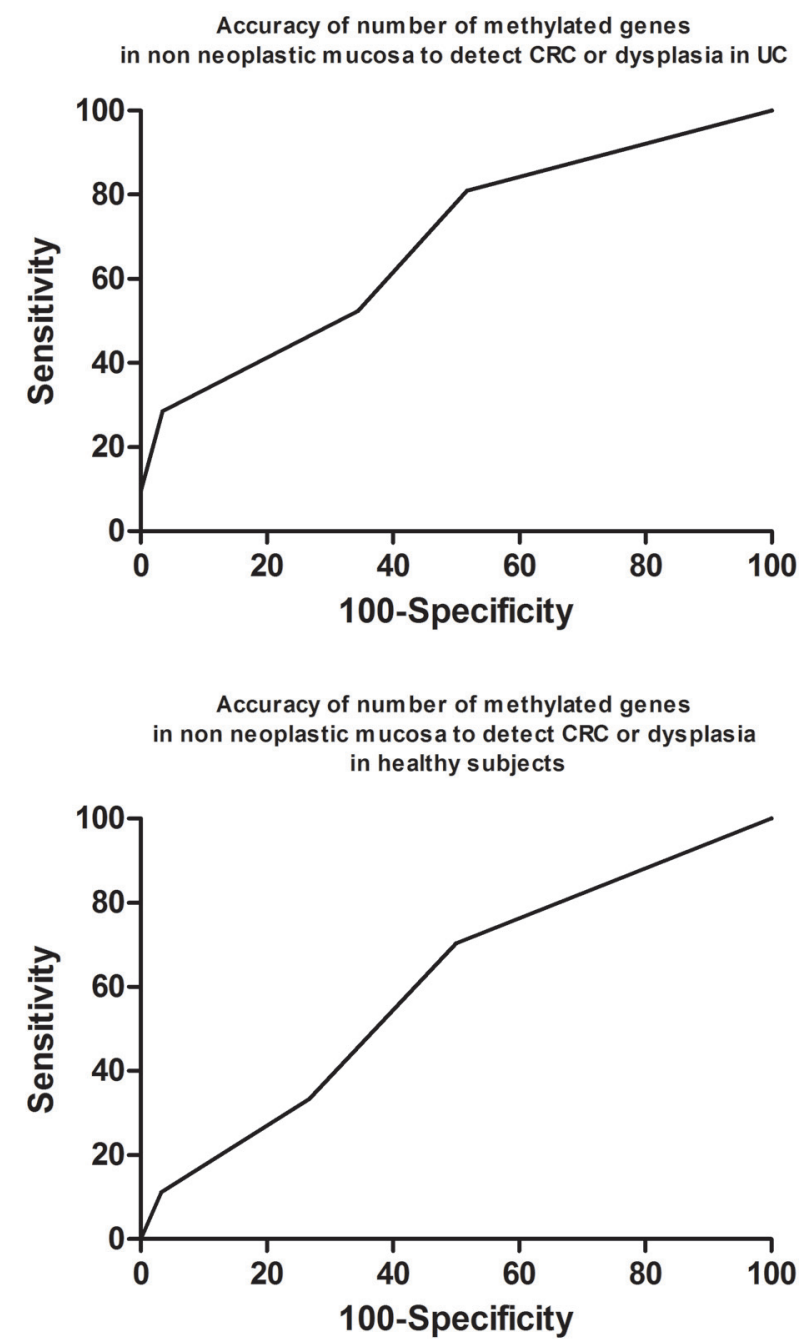

risk approximately $8 \%$ twenty years after the diagnosis $[2,3,4]$. Therefore, patients with UC at moderate or high risk for $\mathrm{CRC}$ are advised to undergo frequent surveillance colonoscopy with multiple random biopsies [8-10]. The identification of markers for UC-related cancer that can be detected in the non neoplastic areas of the colon would greatly simplify the clinical practice and reduce patients' stress [11]. An altered methylation pattern of APC, CDH13, MGMT, MLH1 and RUNX3 genes is a well recognized characteristic of CRC tumor cells [12]. Therefore, the aim of our study is to evaluate the methylation status of these gene in the non-neoplastic colonic mucosa at the different steps of not inflammatory and UC-related carcinogenesis and to investigate the possible role of genomic methylation in the non-neoplastic colonic mucosa as a marker of UC-related CRC.
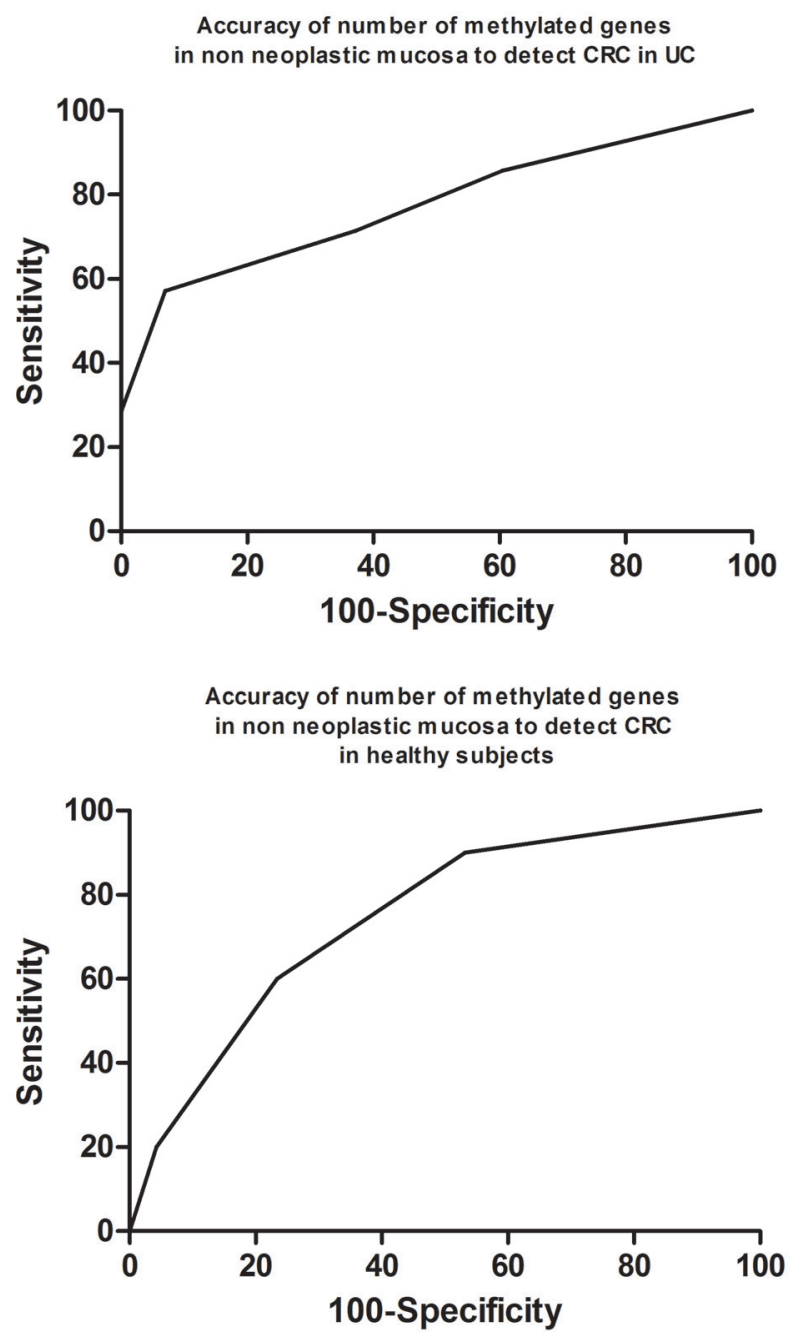

Figure 3: Accuracy of the number of methylated genes in predicting the presence of cancer in the colon. (A) ROC curve showing the accuracy of the number of methylated genes in colonic mucosa without lesions in predicting the presence of dysplasia or cancer in patients UC. (B) ROC curve showing the accuracy of the number of methylated genes in colonic mucosa without lesions in predicting the presence of cancer in patients UC. (C) ROC curve showing the accuracy of the number of methylated genes in colonic mucosa without lesions in predicting the presence of adenoma or cancer in patients without UC. (D) ROC curve showing the accuracy of the number of methylated genes in colonic mucosa without lesions in predicting the presence of cancer in patients without UC. 
Several CpG-island-associated genes involved in cell growth control or metastasis can become hypermethylated and silenced in tumors [25]. Among these APC [13, 26], Cadherin [14, 27], MGMT [15, 28], MLH1 $[16,29]$ and RUNX3 [17]. The main original observation in our study is that these genes may become methylated even in the non-neoplastic mucosa of patients with CRC (UC-related or not). Moreover, some of them, such as APC and RUNX3 were significantly more frequently methylated in patients with UC and cancer compared to patients with non inflammatory adenocarcinoma and CDH13 was more frequently methylated in patients with UC and dysplasia compared to those with adenoma. These observations are very likely due to the higher expression of Dnmts in all the carcinogenesis steps in patients with UC compared to those without inflammation in their colon. During chronic inflammation. intrinsic mediators of inflammatory responses, such as proinflammatory cytokines and reactive oxygen and nitrogen species, can induce genetic and epigenetic modifications, including

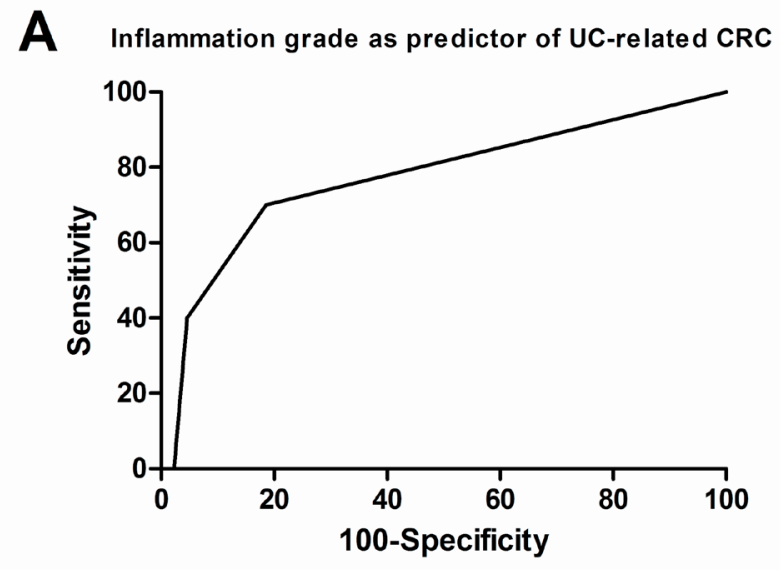

point mutations, deletions, duplications, recombinations, and methylation of some tumor-related genes through various mechanisms.

Dnmt-1, -3a, and -3b mRNA expression was quantified to understand how the inflammatory condition may influence the genomic methylation status in the non-neoplastic colonic mucosa. In our series, the expression of Dnmts 1 and 3A showed a significant higher expression in each steps of the UC-related carcinogenesis compared to the non-inflammatory corresponding steps. Although the mechanism responsible for increased expression of DNMT1 in UC is not well known, a previous report of DNMT1 protein overexpression in bladder cancer suggested that the mRNA expressions of DNMT1 we observed in UC may be attributable to increased cell proliferation caused by inflammation [23-26].

In fact, in our series, in non-neoplastic colonic mucosa, the number of methylated genes resulted significantly higher in patients with CRC and in those with UC-related CRC compared to the $\mathrm{HC}$ and UC

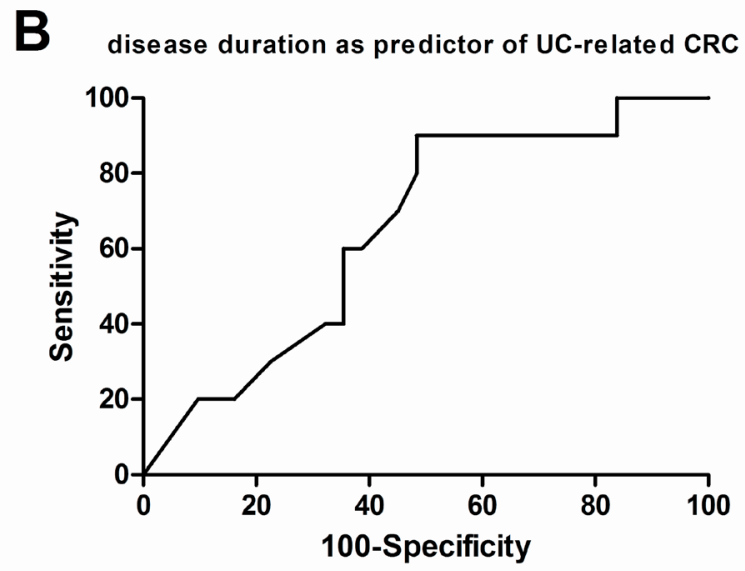

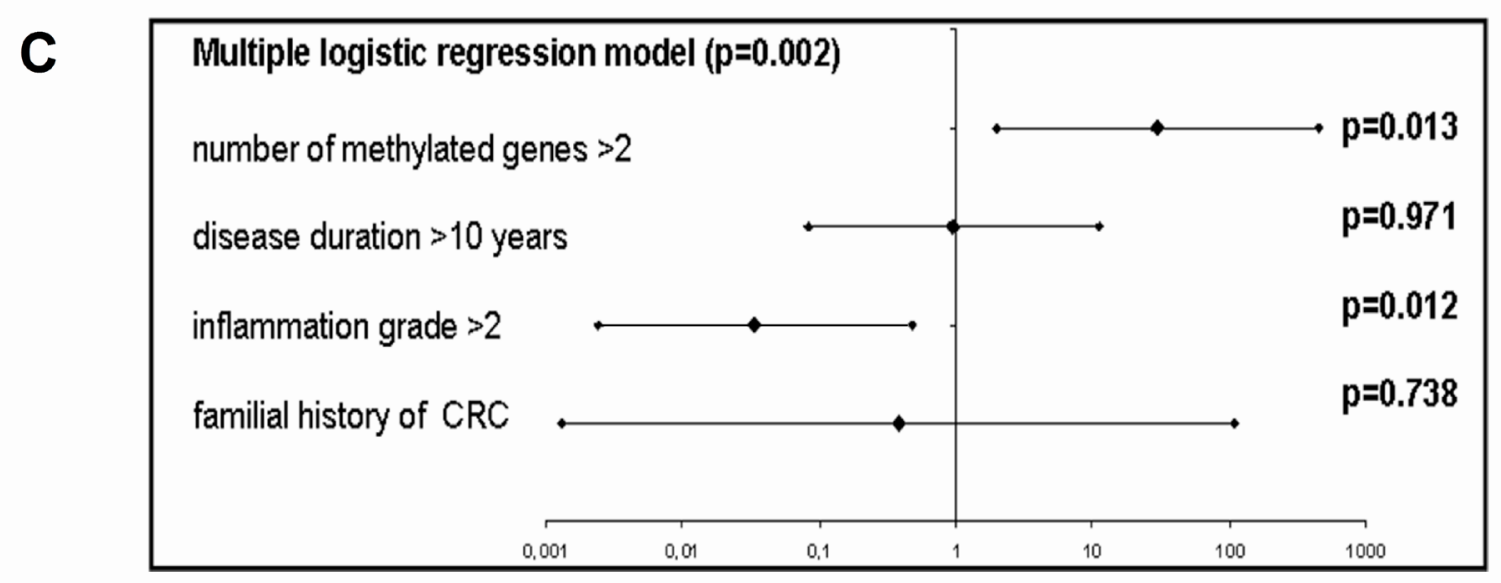

Figure 4: Number of methylated genes as risk factor for CRC in UC. (A) ROC curve showing the accuracy of inflammatory grade as measured by Floren score in predicting UC-related CRC. (B) ROC curve showing the accuracy of disease duration of patients in predicting UC-related CRC. (C) Forrest plot showing a multiple logistic regression analysis including Floren score, methylation score, disease duration and familial history of CRC as possible predictors of UC-related CRC. 
patients and patients with dysplastic lesion of the colon. This observation has two direct implications. The first one is that in patients with high DNMTs activity, such as patients with UC, possible inhibitory agents might be tested to reduce the risk of CRC onset. The second and more direct one is that the number of methylated genes in non-neoplastic mucosa may be used in the CRC surveillance in UC patients. In fact, according to some investigators, methylation of some genes such as RUNX3, MINT1, and COX-2 can be considered potential biomarkers to detect signs of CRC in samples taken from inflamed areas of colon in UC patients [11]. Therefore, we assessed the accuracy of the number of methylated genes as predictor of CRC in inflamed and in normal condition and we concluded that either in UC or in not inflammatory condition the number of methylated genes in colonic mucosa without lesions predicted the presence of CRC with good accuracy. These data suggested a practical application for UC patients since in their case CRC surveillance is currently conducted with regular colonoscopies [30] while for healthy subjects CRC screening program is currently based on fecal blood test. In UC patients a simple sigmoidoscopy might provide adequate mucosal samples for assessing CRC risk with the advantage of a much easier bowel preparation and a shorter and less difficult endoscopic exam. Furthermore, the test has a high specificity (that measures the proportion of negatives that are correctly identified as such) making it an ideal first line test (true positive may be later assessed at second line test, in this case by a full colonoscopy with random biopsies). Indeed, the accuracy of the test might be improved with the histological grade of inflammation on the same mucosa sample as shown by multivariate analysis. Finally a further step in the clinical application of these observation might be the research of the methylation status of these genes in the stools. In fact, methylation of BMP3, vimentin, EYA4 and NDRG4 has been found to be highly discriminant in detecting signs of CRC in stool specimens [31].

In conclusions, although further longitudinal studies with large numbers of subjects are needed to clarify the role of methylation status of APC, CDH13, MGMT, MLH1 and RUNX3 in non-neoplastic mucosa as a marker of UC-associated CRC, and to validate the predictive power and clinical value of analysis of the number of methylated genes, these preliminary results could allow for the adjustment of a patient's surveillance interval and to select UC patients who should undergo intensive surveillance.

\section{MATERIALS AND METHODS}

\section{Patients}

A prospective cohort study of healthy controls $(n=30)$ and patients $(n=77)$ who underwent colonoscopy for screening or post-operative follow-up, colonic resection for colorectal cancer, or restorative proctocolectomy for ulcerative colitis (UC) was designed. No patients was also affected by primary sclerosing cholangitis. Biopsy samples of healthy mucosa $(n=30)$, of normal and diseased mucosa from patients with dysplastic adenoma $(n=14)$, and from patients with colon adenocarcinoma $(n=10)$ were collected. Biopsy samples of the diseased mucosa of patients with UC $(n=29)$, of those with UC and dysplasia $(n=14)$, and of those with UC and cancer $(n=10)$ were also collected. The study, which received institutional review board (Ethical Committee of the Veneto Institute of Oncology) approval (project MICCE1 IOV 2011/53), was performed according to the principles of the Declaration of Helsinki, and all those participating signed informed consent forms.

\section{Histopathology}

Sections ( $3 \mu \mathrm{m})$ from formalin-fixed and paraffinembedded specimens were stained with hematoxylineosin. Histological inflammation was quantified and classified using Floren's score [18] while the Vienna classification of gastrointestinal epithelial neoplasia was used to classify the different step of the colonic carcinogenesis [19].

\section{Gene expression analysis}

Total RNA was extracted using the RNeasy Plus Kit (Qiagen) according to the manufacturer's protocol. At that point $0.5 \mu \mathrm{g}$ total RNA was converted to cDNA using the Applied Biosystems cDNA Synthesis kit, again, according to the manufacturer's instructions. DNA methyltransferase-1, -3a, -3b (Dnmt-) mRNA expression was quantified by real time qRT-PCR. Specific mRNA transcripts were quantified with SYBR Green PCR Master Mix in a ABI PRISM 7000 Sequence Detection System (Applied Biosystems). Actab expression was used as reference gene for normalization. Primer sequences and PCR conditions are outlined in Table 1.

\section{Methylation specific PCR}

Genomic DNA was extracted from tissues using a DNeasy Blood \& Tissue Kit (Qiagen) according to the manufacturer's directions. Sodium bisulfate modification of gDNA was performed using the EZ DNA MethylationGold Kit (Zymo Research) following the manufacturer's instructions. The primers for APC, CDH13, MGMT, MLH1 and RUNX3 methylation-specific PCR and PCR conditions are outlined in Table 2. The EpiTect PCR Control DNA Set (Qiagen) was used as the positive control for the methylated and unmethylated genes. Each PCR was done in a final volume of $25 \mu \mathrm{L}$ containing $10 \mathrm{ng}$ of bisulfite converted gDNA, $1 \times$ PCR buffer, $0.25 \mathrm{mmol} / \mathrm{L}$ deoxynucleotide triphosphate, $400 \mathrm{nmol} / \mathrm{L}$ 
Table 2: Patients characteristics

\begin{tabular}{|c|c|c|c|}
\hline $\begin{array}{l}\text { Non-inflammatory } \\
\text { carcinogenesis }\end{array}$ & $\begin{array}{l}\text { Healthy } \\
\text { controls }\end{array}$ & $\begin{array}{c}\text { Adenoma } \\
\text { and dysplasia }\end{array}$ & Cancer \\
\hline Patients (n) & 30 & 14 & 10 \\
\hline median age (range) & $59.5(52-69)$ years & $61(51-69)$ years & $63(49-74)$ years \\
\hline gender (male/female) & $14: 16$ & $7: 7$ & $7: 3$ \\
\hline \multirow[t]{2}{*}{ procedures } & colonoscopy: 30 & colonoscopy: 12 & colonic resection: 8 \\
\hline & & RPC: 2 & rectal resection: 2 \\
\hline \multirow[t]{5}{*}{ carcinogenesis stage } & NA & LGD: 9 & T1N0M0: 1 \\
\hline & & HGD: 5 & T2N0M0: 2 \\
\hline & & & T3N0M0: 2 \\
\hline & & & T3N1M0: 4 \\
\hline & & & T3N1M1: 1 \\
\hline UC-related carcinogenesis & $\mathbf{U C}$ & $\begin{array}{c}\mathrm{UC} \\
\text { and dysplasia }\end{array}$ & $\begin{array}{c}\mathrm{UC} \\
\text { and cancer }\end{array}$ \\
\hline Patients (n) & 29 & 14 & 10 \\
\hline median age (range) & $44(16-70)$ years & $55(24-72)$ years & $54(33-67)$ years \\
\hline gender (male/female) & $15: 14$ & $7: 7$ & $6: 4$ \\
\hline \multirow[t]{2}{*}{ procedures } & RPC: 29 & RPC: 14 & RPC: 9 \\
\hline & & & proctectomy: 1 \\
\hline \multirow[t]{3}{*}{ carcinogenesis stage } & NA & LGD: 10 & T1N0M0: 8 \\
\hline & & HGD: 4 & T2N0M0: 1 \\
\hline & & & T4N1M1: 1 \\
\hline
\end{tabular}

HGD. high grade dysplasia; LGD. low grade dysplasia; RPC. restorative proctocolectomy

each primer, and 1 unit ZymoTaq (Zymo Research). PCR amplification was done as follows: $95^{\circ} \mathrm{C}$ for 10 min followed by 40 cycles at $95^{\circ} \mathrm{C}$ for $30 \mathrm{~s}$, the specific annealing temperature for each gene for $30 \mathrm{~s}$ and $72^{\circ} \mathrm{C}$ for $30 \mathrm{~s}$; and, in a final extension step, at $72^{\circ} \mathrm{C}$ for $7 \mathrm{~min}$. PCR products were resolved by $3 \%$ agarose gel electrophoresis and each case was scored as methylated or unmethylated. Since we aimed to investigate the role of methylation we restrictively considered promoter methylation present only if the ratio between methylated/unmethylated band signal was $>1$. The patients' global methylation scores were calculated by summing the number of methylated genes (range 0-5).

\section{Statistical analysis}

Data are shown as mean \pm SEM. Non-parametric Mann-Whitney's $U$-test was carried out to compare independent variables, the Wilcoxon test was used to compare matched variables, and the Kruskal-Wallis ANOVA was performed to compare multiple variables. Receiving operator characteristics (ROC) were assessed by curve analysis as described by Henderson (21). Logistic regression modeling was undertaken to determine whether the grade of histological inflammation as measured with Floren score and the number of methylated genes were independent predictor of UC-related CRC. Both score were dichotomized on the threshold values obtained at ROC curve analysis. Statistical significance was indicated by $p<0.05$.

\section{ACKNOWLEDGMENTS}

The authors are grateful to Mrs. Christina Drace for her assistance in editing the final version of this manuscript.

\section{FUNDING}

This study was partially funded by the Italian Gastroenterological Society (SIGE) POST DOC 2007 AWARD assigned to Marco Scarpa; other sources were Current Research Funding from the Veneto Institute of 
Oncology (IOV-IRCCS) and the Finalized Research (project MICCE1) from the Veneto Region, both assigned to Carlo Castoro.

\section{Authors'contributions}

Marco Scarpa, Melania Scarpa and Ignazio Castagliuolo have made substantial contributions to conception and design and analysis and interpretation of data; has been involved in drafting the manuscript; and has given final approval of the version to be published. Francesca Erroi, Andromachi Kotsafti, Silvia Basato, Paola Brun, Renata D'Incà and Massimo Rugge has made substantial contributions to acquisition of data; has been involved in revising the manuscript critically for important intellectual content; and has given final approval of the version to be published. Imerio Angriman and Carlo Castoro has made substantial contributions to conception and design; has been involved in revising the manuscript critically for important intellectual content; and has given final approval of the version to be published.

\section{Abbreviations}

APC, Adenomatous Polyposis Coli; CDH13, H-cadherin; CRC, colorectal cancer; DNMT, DNA methyltransferase; MGMT, O-6-methylguanine-DNA methyltransferase; MLH1, MutL homolog 1; RUNX3, Runt-related transcription factor 3; UC, Ulcerative Colitis.

\section{CONFLICTS OF INTEREST}

The authors have no conflicts of interest.

\section{REFERENCES}

1. Munkholm P. The incidence and prevalence of colorectal cancer in inflammatory bowel disease. Aliment Pharmacol Ther. $2003 ; 18: 1-5$.

2. Eaden JA, Abrams KR, Mayberry JF. The risk of colorectal cancer in ulcerative colitis: a meta-analysis. Gut. 2001; 48:526-535.

3. Lim $\mathrm{CH}$, et al. Ten year follow up of ulcerative colitis patients with and without low grade dysplasia. Gut. 2003; 52:1127-1132.

4. Lynch DA, Lobo AJ, Sobala GM, Dixon MF, Axon AT, Failure of colonoscopic surveillance in ulcerative colitis. Gut. 1993; 34:1075-1080.

5. Eaden JA, Abrams KR, Mayberry JF. The risk of colorectal cancer in ulcerative colitis: a meta-analysis. Gut. 2001; 48:526-35.

6. Lutgens MW, Vleggaar FP, Schipper ME, et al. High frequency of early colorectal cancer in inflammatory bowel disease. Gut 2008;57:1246-51.
7. Itzkowitz S. Colon carcinogenesis in inflammatory bowel disease. J Clin Gastroenterol. 2003; 36:S70-4.

8. Provenzale D, Onken J. Surveillance issues in inflammatory bowel disease: ulcerative colitis. J Clin Gastroenterol. 2001; 32:99-105.

9. Itzkowitz SH, Present DH. Consensus conference: colorectal cancer screening and surveillance in infl ammatory bowel disease . Inflamm Bowel Dis. 2005; 11:314-21 .

10. Mowat C, Cole A, Windsor A, Ahmad T, Arnott I, Driscoll R, Mitton S, Orchard T, Rutter M, Younge L, Lees C, Ho GT, Satsangi J, et al. IBD Section of the British Society of Gastroenterology. Guidelines for the management of inflammatory bowel disease in adults. Gut. 2011; 60: 571-607. doi: 10.1136/gut.2010.224154.

11. Garrity-Park MM, Loftus EV, Jr, Sandborn WJ, Bryant SC, Smyrk TC. Methylation status of genes in non-neoplastic mucosa from patients with ulcerative colitis-associated colorectal cancer. Am J Gastroenterol. 2010; 105:1610-9.

12. Lao VV, Grady WM, Epigenetics and colorectal cancer. Nat Rev Gastroenterol Hepatol. 2011; 8:686-700.

13. Toyooka S, Toyooka KO, Harada K, et al. Aberrant methylation of the CDH13 (H-cadherin) promoter region in colorectal cancers and adenomas. Cancer Res. 2002; 62:3382-6.

14. Esteller M, Sparks A, Toyota M, et al. Analysis of adenomatous polyposis coli promoter hypermethylation in human cancer. Cancer Res. 2000; 60:4366-71.

15. Esteller M, Garcia-Foncillas J, Andion E, et al. Inactivation of the DNA-repair gene MGMT and the clinical response of gliomas to alkylating agents. N Engl J Med. 2000; $343: 1350-4$

16. 12 Herman JG, Umar A, Polyak K, et al. Incidence and functional consequences of hMLH1 promoter hypermethylation in colorectal carcinoma. Proc Natl Acad Sci USA. 1998; 95:6870-5.

17. Li QL, Ito K, Sakakura C, et al. Causal relationship between the loss of RUNX3 expression and gastric cancer. Cell. 2002; 109:113-24.

18. Robertson KD. DNA methylation, methyltransferases, and cancer. Oncogene. 200; 1 20:3139-3155

19. Hiltunen MO, Alhonen L, Koistinaho J, Myohanen S, aakkonen M, Marin S, Kosma VM, Janne J. Int. J. Cancer. 1997; 70:644-648.

20. Graff JR, Herman JG, Lapidus RG, Chopra H, Xu R, Jarrard DF, Isaacs WB, Pitha PM, Davidson NE, Baylin SB. Cancer Res. 1995; 55:5195-5199.

21. Esteller M, Avizienyte E, Corn PG, Lothe RA, Baylin SB, Aaltonen LA, Herman JG. Epigenetic inactivation of LKB1 in primary tumors associated with the Peutz-Jeghers syndrome. Oncogene. 2000; 19:164-8.

22. Herman JG, Umar A, Polyak K, Gra€ JR, Ahuja N, Issa J-PJ, Markowitz S, Willson JKV, Hamilton SR, Kinzler KW, Kane MF, Kolodner RD, Vogelstein B, et al. Proc. Natl. Acad. Sci. USA. 1998; 98, 6870-6875. 
23. Robertson KD, Uzvolgyi E, Liang G, Talmadge C, Sumegi J, Gonzales FA, et al. The human DNAmethyltransferases (DNMTs) 1,3a and 3b: coordinate mRNA expression in normal tissues and overexpression in tumors. Nucleic Acids Res. 1999; 27:2291-8.doi:10.1093/nar/27.11.2291

24. Nakagawa T, Kanai Y, Saito Y, Kitamura T, Kakizoe T, Hirohashi S. Increased DNA methyltransferase-1 protein expression in human transitional cell carcinoma of the bladder. J Urol. 2003; 170:2463-2466.

25. Fujii S, Katake Y, Tanaka H. Increased expression of DNA methyltransferase-1 in non-neoplastic epithelium helps predict colorectal neoplasia risk in ulcerative colitis. Digestion. 2010; 82:179-86. doi: 10.1159/000311064.

26. Subramaniam D, Thombre R, Dhar A, Anant S. DNA methyltransferases: a novel target for prevention and therapy. Front Oncol. 2014 May 1; 4:80. doi:10.3389/ fonc. 2014.00080 .

27. Øresland T, Bemelman WA, Sampietro GM, Spinelli A, Windsor A, Ferrante M, Marteau P, Zmora O, Kotze PG, Espin-Basany E, Tiret E, Sica G, Panis Y, et al. European Crohn's and Colitis Organisation (ECCO). European evidence based consensus on surgery for ulcerative colitis. J Crohns Colitis. 2015 Jan; 9:4-25. doi: 10.1016/j. crohns.2014.08.012.

28. Kisiel JB, Yab TC, Nazer Hussain FT, Taylor WR, GarrityPark MM, Sandborn WJ, Loftus EV, Wolff BG, Smyrk TC, Itzkowitz SH, Rubin DT, Zou H, Mahoney DW, et al. Stool DNA testing for the detection of colorectal neoplasia in patients with inflammatory bowel disease. Aliment Pharmacol Ther. 2013 Mar; 37:546-54. doi: 10.1111/apt.12218.

29. Florén $\mathrm{CH}$, Benoni C, Willén R. Histologic and colonoscopic assessment of disease extension in ulcerative colitis. Scand J Gastroenterol. 1987 May; 22:459-62.

30. Schlemper RJ, Riddell RH, Kato Y, Borchard F, Cooper HS, Dawsey SM, Dixon MF, Fenoglio-Preiser CM, Fléjou JF, Geboes K, Hattori T, Hirota T, Itabashi M, et al. The Vienna classification of gastrointestinal epithelial neoplasia. Gut. 2000 Aug; 47:251-5.

31. Henderson AR. Assessing test accuracy on its clinical consequence: a primer for receiver operative characteristics curve analysis. Ann Clin Biochem. 1993; 30:521-539. 